\title{
Clonal diversity in the ancient asexual ostracod Darwinula stevensoni assessed by RAPD-PCR
}

\author{
K Van Doninck ${ }^{1,2}$, I Schön ${ }^{1}$, K Martens ${ }^{1,3}$ and T Backeljau ${ }^{1,4}$ \\ ${ }^{1}$ Freshwater Biology and Malacology Sections, Royal Belgian Institute of Natural Sciences, Vautierstraat 29, B-1000 Brussels, Belgium; \\ ${ }^{2}$ Laboratory of Cell Genetics, Free University of Brussels, Pleinlaan 2, B-1050 Brussels, Belgium; ${ }^{3}$ University of Ghent, Vakgroep \\ Biologie, KL Ledeganckstraat 35, B-9000 Ghent, Belgium; ${ }^{4}$ Evolutionary Biology group, University of Antwerp, Groenenborgerlaan 171, \\ B-2020 Antwerp, Belgium
}

As Darwinulidae (Ostracoda) are considered to be ancient asexuals with a wide geographical and ecological distribution, they are expected to have accumulated mutations during a long timeframe. However, previous studies on genetic variability suggested a low genetic divergence within the darwinulid species Darwinula stevensoni.

Here, overall genotopic diversity of $D$. stevensoni is estimated with the Random Amplified Polymorphic DNA (RAPD) technique. Using six primers revealing 47 consistently scorable polymorphic loci, substantial clonal diversity within this species is detected. Five of the seven surveyed populations are multiclonal. Moreover, the seven populations have a different clonal composition with almost all of the observed clonal genotypes being restricted to single populations, indicating the absence of a single widespread 'clone'.

The observed clonal diversity seems to refute the existence of a widespread general purpose genotype for $D$. stevensoni. However, in light of previously detected uniformity at functional loci, we reconsider the definition of a GPG. We suggest that it need not imply a genome-wide fixed genotype, but rather consists of a set of ecologically relevant genes.

Heredity (2004) 93, 154-160, advance online publication, 26 May 2004; doi:10.1038/sj.hdy.6800486

Keywords: RAPD; genetic variation; clone; ancient asexual; Darwinula stevensoni; general purpose genotype

\section{Introduction}

The Ostracoda are a unique animal group in which an extensive range of reproductive strategies occurs together with a high incidence of transitions from sexual to asexual reproduction (Martens, 1998). Allozyme studies of mother-daughter pedigrees have provided some evidence that the latter occurs apomictically (see Chaplin, 1992; Havel and Hebert, 1993; Rossi et al, 1993).

The classical notion that asexual organisms have low levels of genetic variation turns out to be the exception rather than the rule, and depends on clonal ancestry (Parker, 1979), the amount of recurrent hybridisation with sexual relatives (Lynch et al, 1989), automictic events (Little and Hebert, 1994) and the frequency of mixis in the life cycle (Hughes, 1989; Carvalho, 1994). Most asexual ostracod populations hitherto studied belong to species with mixed reproduction and show high clonal diversities (reviewed in Havel and Hebert, 1993; Rossi et al, 1998). Some of this clonal variation has undoubtedly arisen from mutations, but the high levels are mainly the result of repeated clonal origins from bisexual conspecifics and/or occasional hybridisation between asexual females and males (Chaplin et al, 1994; Little and Hebert, 1994; Schön et al, 2000 and references therein). The joint occurrence of sexual and asexual taxa

Correspondence: K Van Doninck. Current address: Harvard University, 7 Divinity Avenue, Cambridge, MA 02138, USA.

E-mail:kvdoninc@mcb.harvard.edu

Received 2 June 2003; accepted 26 March 2004; published online 26 May 2004 in some genera suggests that asexual ostracods evolved recently (Chaplin et al, 1994). However, the family Darwinulidae seems to provide a striking exception to this latter pattern. Darwinulids are considered to be ancient asexuals because their fossil record shows a complete absence of males since the Triassic, that is, during the last 200 million years (Martens et al, 2003). The most common species of this family, Darwinula stevensoni, is assumed to have reproduced apomictically (Butlin et al, 1998) for at least 25 myr (Straub, 1952). As this species has no sexual relatives, interbreeding and recurrent origins of clones could not have generated clonal diversity, so that new mutations are probably the only source of variation. Hence, when this process operates over long timeframes, genetic differences are expected to continuously accumulate between clonal lineages (Butlin et al, 1998) and, since recombination is absent, also between alleles within lineages (Mark Welch and Meselson, 2000). In contrast with these expectations, little genetic variation has thus far been found within $D$. stevensoni by allozyme screening and DNA sequencing (see Havel and Hebert, 1993; Rossi et al, 1998; Schön et al, 1998; Schön and Martens, 2003). This apparently low genetic variability is intriguing considering the wide geographical and ecological distribution of D. stevensoni (Rossetti and Martens, 1998).

Generally, the widespread distribution of asexual lineages is explained either through dispersal of a single clonal lineage with wide ecological tolerance (ie general purpose genotypes or GPG) or by the presence of many locally adapted clones. Circumstantial evidence for the wide ecological tolerance of asexual species was 
provided by the broad geographical distribution of a number of asexuals compared to their sexual relatives (Lynch, 1984; Parker and Niklasson, 1998 and references therein). Vrijenhoek (1998) however asserted that molecular markers should be used in order to investigate whether a widespread asexual taxon is composed of a single plastic clonal lineage (a GPG) or of several ecologically specialised clones. Understanding the geographic distribution of asexuals can thus be partly achieved through a combined analysis of their population genetics and environmental tolerance (Jacobsen et al, 1996; Vrijenhoek, 1998).

Recently, Van Doninck et al (2002) showed that D. stevensoni had a higher tolerance for salinity and temperature than other asexual ostracods, while Rossi et al (2002) reported a high hypoxia tolerance for $D$. stevensoni. These results suggest that $D$. stevensoni shows the ecological characteristics of a GPG. However, although current genetic data from $D$. stevensoni, using several genetic markers, seem to suggest that these ecological characteristic are associated with a low genetic variability (Rossi et al, 1998; Schön et al, 1998; Schön and Martens, 2003), overall genotypic diversity in this species remains unknown and needs investigation.

Therefore, the aim of the present study is to estimate clonal diversity in $D$. stevensoni from different geographic populations using genome-wide genetic markers. In this way, this study investigates (1) the degree of clonal similarity within and between $D$. stevensoni populations, (2) to what extent $D$. stevensoni populations are dominated by one or a few widespread clonal genotypes and (3) whether the D. stevensoni populations of the ecological study of Van Doninck et al (2002) are monoclonal and genetically identical. To achieve these goals, the RAPDPCR priming technique was applied (Williams et al, 1990).

\section{Materials and methods}

\section{Sample collection}

Seven populations of D. stevensoni were surveyed by means of RAPD-PCR (Table 1), three of which BH, FC and IB were also included in previous ecological tests and were then labelled DsB, DsF and DsI, respectively (Van Doninck et al, 2002). After field collection, specimens were maintained alive in the laboratory in plastic sampling bottles with sediment and water of the sampling location. Bottles were continuously oxygenated and distilled water was added to compensate for evaporation. Prior to DNA extraction, individuals were starved for at least $48 \mathrm{~h}$ in distilled water, transferred individually to $1.5 \mathrm{ml}$ eppendorf tubes and stored at $-80^{\circ} \mathrm{C}$.

\section{DNA extraction}

Four different methods to extract DNA from individual ostracods were compared, viz: (1) Chelex extraction according to Schön et al (1998), (2) the CTAB protocol used for mites (Van Opijnen and Breeuwer, 1999), (3) the Gene Releaser protocol of Schizas et al (1997) (for harpacticoid copepods) and Oakley (pers. comm.) (for ostracods), and (4) the protocol implemented by QIAamp DNA Mini kit (Qiagen) for extractions of tissues, using $50 \mu \mathrm{l}$ DNA elution buffer instead of $200 \mu \mathrm{l}$ in the last two steps. Extracted DNA was stored at $-20^{\circ} \mathrm{C}$.

\section{Optimisation of RAPD procedure}

To minimise experimental errors causing artifactual RAPD bands, the amplification protocols were optimised and standardised so that bands were consistent across replicates (see Black IV, 1993; Guirao et al, 1995). To this end, a series of optimisation experiments were conducted in which concentrations of DNA template, primers, nucleotides, $\mathrm{MgCl}_{2}$ and Taq polymerase were varied to determine the conditions yielding the most intense banding patterns. The optimised reaction conditions were as follows: total reaction volumes of $25 \mu \mathrm{l}$ were used with final concentrations of $50 \mathrm{mM} \mathrm{KCl}, 10 \mathrm{mM}$ Tris- $\mathrm{HCl}$, pH 9.0, 2 mM MgCl $2,100 \mu \mathrm{M}$ dNTP (Promega), $0.2 \mu \mathrm{M}$ primer, $0.5 \mathrm{U}$ Taq polymerase (Pharmacia) and $2 \mu \mathrm{l}$ DNA from a stock solution of $100 \mu \mathrm{l}$. Amplifications were conducted in a Biometra (Tgradient) Thermal Cycler (Westburg) with the following settings: $5 \mathrm{~min}$ denaturation at $95^{\circ} \mathrm{C}$, followed by 40 cycles of $1 \mathrm{~min}$ at $95^{\circ} \mathrm{C}$, annealing at $37^{\circ} \mathrm{C}$ for $1 \mathrm{~min}$ and elongation at $72^{\circ} \mathrm{C}$ for $2 \mathrm{~min}$. An additional $10 \mathrm{~min}$ elongation at $72^{\circ} \mathrm{C}$ followed the last cycle. Amplification products were electrophoresed on $6 \%$ polyacrylamide gels at $180 \mathrm{~V}$ for $5 \mathrm{~h}$ in $1 \times$ TBE buffer (1 M Tris, $0.83 \mathrm{M}$ Boric Acid, $10 \mathrm{mM}$ EDTA) together with a $100 \mathrm{bp}$ ladder (Pharmacia). After electrophoresis, gels were silver stained. Agarose gels, stained with ethidium bromide, were also used for electrophoresis during optimisation.

A prescreening with 52 primers (six from Pharmacia and 46 from Operon primer kits OPA, OPB and OPE) was also conducted, using one to three individuals of each population. Only those primers that yielded positive amplifications and revealed well visible RAPD fragments for all populations were used for further study. Next, those selected primers were tested in triplicate to investigate their reproducibility; that is using

Table 1 Populations of D. stevensoni used in RAPD analysis

\begin{tabular}{lllllll}
\hline Code & \multicolumn{1}{c}{ Site } & $n$ & $N_{c}$ & $N_{c} / n$ & $G_{0}$ & \multicolumn{1}{c}{$G_{0} / N_{c}$} \\
\hline BM & Grand Mellaerts, Belgium & 20 & 5 & 0.25 & 3.52 & 0.70 \\
BH & Hollandersgatkreek, Belgium & 21 & 1 & 0.048 & 1 & 0.086 \\
BT & Tenreuken, Belgium & 25 & 8 & 0.32 & 3.93 & 0.491 \\
FC & Clue de la Fou, France & 17 & 1 & 0.059 & 1 & 0.123 \\
IB & Lough Ballyquirke, Ireland & 21 & 2 & 0.095 & 1.6 & 0.8 \\
IL & Lough Lickeen, Ireland & 12 & 2 & 0.167 & 1.8 & 0.9 \\
SC & Caveta, Spain & 25 & 14 & 0.56 & 7.53 & 0.005 \\
\end{tabular}

The sample size per population $(n)$, number of clones $\left(N_{c}\right)$, three measures of genotypic diversity $\left(N_{c} / n, G_{0}\right.$ and $\left.G_{0} / N_{c}\right)$ and Nei's $(1973)$ gene diversity $(h)$ are given for each tested population. 
the same primers, the same DNA stock (from individuals of the seven populations) and the same batch of reagents, three independent PCRs were conducted, electrophoresed on different gels and compared to investigate whether that primer revealed polymorphic, reproducible bands for each tested population. Six primers, showing $100 \%$ reproducibility of distinct bands, were retained and used to analyse variation in $D$. stevensoni, viz OPA-2 (TGCCGAGCTG), OPA-8 (GTGACGTAGG), OPA-10 (GT GATCGCAG), OPB-7 (GGTGACGCAG), OPB-10 (CTGC TGGGAC) and OPE-4 (GTGACATGCC).

In order to examine whether epibionts on carapaces of specimens could affect the RAPD banding patterns, DNA was extracted only from the bodies of at least three individuals from each population and analysed in the optimisation phase.

\section{Study of genetic variation}

For the experiment, at least 20 individuals of each $D$. stevensoni population were analysed, except for populations IL (12) and FC (17) (Table 1). All RAPD-PCR reactions were repeated twice: using the same primers and individuals, the replicate PCRs were conducted independently on different days. Samples from previous amplifications were included in all gel runs to ensure comparability of band profiles. Furthermore, for each primer, all different genotypes detected in the seven populations were re-evaluated by running them simultaneously on single gels. Only polymorphic bands that were distinct, intense and completely reproducible were scored (presence/absence: p/a) (Figure 1). Multilocus genotypes were then defined by combining $\mathrm{p} / \mathrm{a}$ data for the scored polymorphic RAPD bands. Thus, genotypes differing by a single band from each other were considered to be different clonal genotypes. Negative controls were included in each PCR reaction to ensure that all RAPD bands resulted from template DNA only.

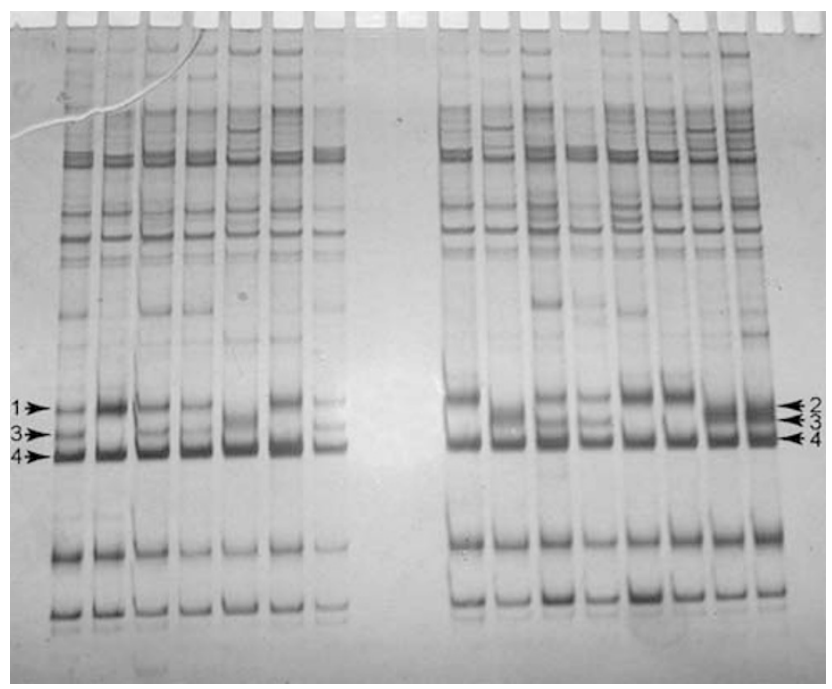

Figure 1 RAPD profiles of D. stevensoni from Grand Mellaerts Belgium (BM) amplified with primer OPA-2. The lanes indicated by an arrow and numbered were the distinct, polymorphic bands that were scored for this specific population and primer; the other bands present on the gel were not scored because they were not $100 \%$ reproducible. Note that three different genotypes can be inferred from the four scored bands (presence/absence data).
Two components of genetic variation were evaluated. The first component was Nei's (1973) gene diversity $h$ as estimated with the computer program POPGENE v. 1.20 (Yeh and Boyle, 1997). The second component was the clonal diversity, for which three indices were calculated: (1) the proportion of distinguishable clones $N_{\mathrm{c}} / n$ with $N_{\mathrm{c}}$ being the number of clonal genotypes and $n$ the population size; when all individuals have a unique genotype $N_{\mathrm{c}} / n$ equals 1 , whereas $N_{\mathrm{c}} / n$ approaches 0 when there are few clonal genotypes (Chen et al, 2002); (2) the observed genotypic diversity $G_{0}=1 /\left(\sum_{i}^{k} g_{i}^{2}\right)$ where $g_{i}$ is the frequency of the $i$ th genotype (or clone) and $k$ the total number of genotypes (Stoddart, 1983); $G_{0}$ varies from a minimum of 1 , when there is a single genotype, to a maximum of $k$, when genotypes are evenly distributed; (3) the genotypic evenness (ie the number of individuals per clonal genotype), $G_{0} / N_{c}$, has a maximum value of 1 when all individuals are distributed evenly among the clones and will approach 0 if a single clone dominates the population (Coffroth and Lasker, 1998). For comparison, these indices were also calculated from asexual populations of other species for which sufficient multilocus data from allozyme, RAPD or microsatellite analyses were available (the complete review with the calculated population parameters is available from KVD upon request).

Band similarities between multilocus genotypes were inferred using the Nei and Li (1979) coefficient (S) calculated by the NT-SYS v. 1.80 software (Rohlf, 1993), in which this coefficient is referred to as 'Dice coefficient'. $S$-values were subjected to UPGMA clustering using the same program. The stability of the UPGMA topology was assessed via bootstrapping over 1000 replicates using the program WinBoot (Yap and Nelson 1996).

\section{Results}

\section{Reproducibility}

Optimal DNA extractions were achieved with the QIAamp DNA Mini kit, by the criterion that sharper RAPD bands were obtained with this method. In this study, polyacrylamide gels were used instead of agarose gels because more bands per primer were visible and bands were intenser, resulting in a higher resolution and a more accurate scoring (see also Fukatsu and Ishikawa, 1994).

As only those primers revealing a clear, intense banding pattern were selected during the optimisation and a conservative approach was followed in scoring only clear, totally reproducible bands, we encountered no difficulties in obtaining reproducible RAPD profiles. One primer revealed between four and 12 scorable loci (or bands) for all seven populations. Additional bands present on the gel were not scored because they were not distinct or reproducible (see Figure 1). Moreover, the scoring of bands was made directly from the gel. As a result, scored polymorphic bands were identical between replicates of the same individuals (eg Figure 2).

\section{Clonal population structure}

The six RAPD primers yielded 47 consistently scorable, polymorphic bands (or loci), which together defined 33 multilocus genotypes (here further referred to as 'clones') in 141 individuals of seven $D$. stevensoni populations. 
Populations $\mathrm{BH}$ and $\mathrm{FC}$ showed no genotypic variation $\left(N_{\mathrm{c}}=1\right)$. The other populations consisted of several clones $\left(N_{\mathrm{c}}\right.$ ranged from 2 to 14$)$ with most clones (14) being detected in the Spanish population SC (Table 1). Population IL was included in these calculations of genetic variation because it clearly represented another clone. Yet in the calculations of Nei and Li's (1979) coefficient $(S)$ and their UPGMA clustering, IL was excluded because of incomplete data. This latter analysis showed that clones clustered by geographic region (Belgium, France, Ireland and Spain), while no genotype was found in more than one region (Figure 3). In Belgium, where more than one population was sampled,

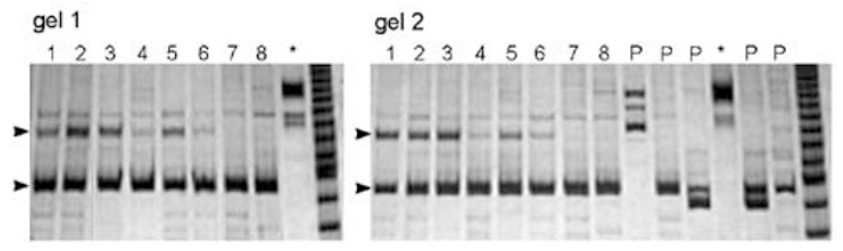

Figure 2 RAPD profiles of D. stevensoni from Tenreuken, Belgium (BT), amplified with primer OPA- 8 . The numbered lanes of gel 1 and gel 2 are replicates from subsequent PCR, each representing an individual. The lane labelled with ${ }^{*}$ is a reference, included in the different gel runs. Lanes labelled with the letter P represent RAPD profiles from other tested populations amplified with OPA-8. The distinct, polymorphic bands scored only for population BT are indicated with an arrow.

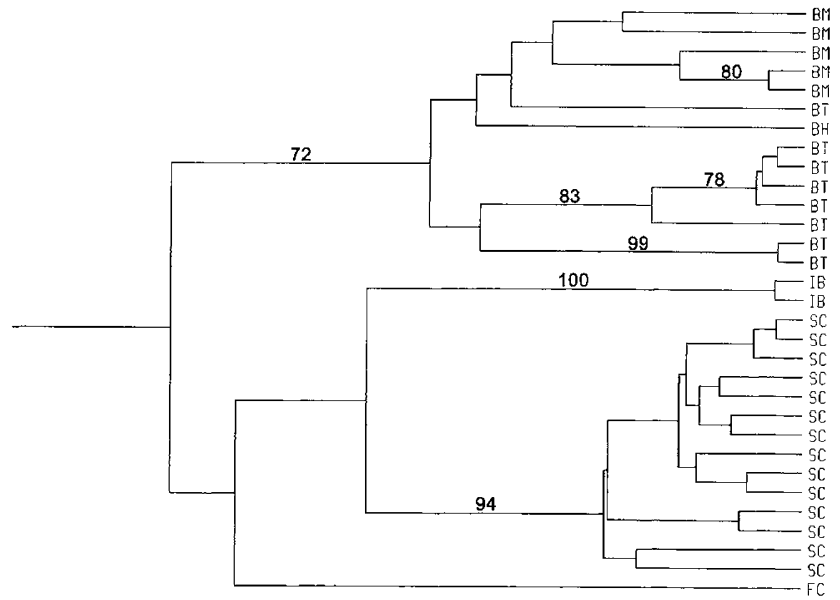

Figure 3 UPGMA dendrogram showing the relationships among $D$. stevensoni populations. Numbers represent bootstrap values (1000 replicates). clones largely cluster by population, but this patterning is not fully bootstrap supported. Moreover, one clone from BT clustered with population BM, though with low bootstrap support (Figure 3).

Within each of the analysed populations, clones differed from each other only by single RAPD bands while most clones from different populations differed by at least 15 bands. In addition, none of the clones was present in more than one population.

\section{Genotypic diversity}

Taken over the seven populations studied here, the observed genotypic diversity $G_{0}$ for $D$. stevensoni varied between 1 and 7.53, the proportion of distinguishable clones $N_{\mathrm{c}} / n$ ranged from 0.048 to 0.56 (Tables 1 and 2).

The populations with the lowest $G_{0}$ and $N_{\mathrm{c}} / n$ are $\mathrm{BH}$ and FC, in which the individuals belong to the same clone. Within each population from Ireland (IB and IL) two clones were found, differing from each other by a single band. Populations BM, BT and SC contained five, eight and 14 clones respectively (Table 1). Evenness, $G_{0} /$ $N_{\mathrm{c}}$, also varied among the seven populations, ranging between 0.491 and 1.000 (Table 1). For the two populations, BT and SC, the numbers of individuals per clone was not evenly distributed $\left(G_{0} / N_{c}<0.55\right.$, Table 1$)$. Among the BT population, two clones were found with eight and nine individuals and five clones with only a single individual. For the Spanish population (SC) we found one clone with seven individuals, 10 clones having one individual and the other clones with four or less individuals.

\section{Relation between ecological tolerance and population genetics}

The three D. stevensoni populations $\mathrm{BH}, \mathrm{FC}$ and IB, which have been tested for their salinity and temperature tolerance (Van Doninck et al, 2002), are genetically different since even the most similar clones from the different populations differ by more than 15 RAPD bands.

\section{Discussion}

\section{Genetic variation in $D$. stevensoni}

Previous surveys of population genetic variation in $D$. stevensoni reported limited allozyme variation with seven different genotypes in 36 populations and $79 \%$ of the individuals belonging to the same genotype (Rossi et al, 1998). However, this result was based on only two enzyme systems of which only Gpil was polymorphic. Yet, using this marker, 14 European populations were multiclonal. Similarly, Havel and Hebert (1993) reported two allozyme clones in a single population from

Table 2 Mean values of $N_{\mathrm{c}}, N_{\mathrm{c}} / n, G_{0}$ and $G_{0} / N_{\mathrm{c}}$ (over seven populations) in D. stevensoni, minimum and maximum values in parentheses

\begin{tabular}{lccccc}
\hline Species & Pop & $N_{c}$ & $N_{c} / n$ & $G_{0}$ & $G_{0} / N_{c}$ \\
\hline Darwinula stevensoni & 7 & 4.7 & 0.214 & 0.776 \\
& & $(1-14)$ & $(0.048-0.56)$ & $(1-7.51)$ & $(0.54-1)$ \\
Asexual species & $1-55$ & $1-8.67$ & $0.04-0.46$ & $1-5.63$ & $0.47-1$ \\
\hline
\end{tabular}

Pop $=$ number of analysed populations. For comparison, ranges of the means of the genotypic diversity measures of other asexual populations are given. 
Northern America using one polymorphic locus, Pgm. Direct DNA sequencing suggested a complete lack of variation in the nuclear ribosomal ITS1 region of D. stevensoni over a geographical range from Finland to South Africa, based on 56 individuals from 19 populations. Some albeit low genetic variation was detected in the mitochondrial COI region (Schön et al, 1998). Recently, Schön and Martens (2003) reported low genetic diversity within and between $D$. stevensoni individuals using three nuclear regions ITS (see also Gandolfi et al, 2001), hsp82 (exon and intron region) and a Calmodulin intron, providing no evidence of the Meselson effect.

In this study, clonal diversity was detected in seven populations of $D$. stevensoni using RAPD-PCR. All of the 33 observed clonal genotypes of $D$. stevensoni were restricted to single populations, indicating the absence of a common widespread (dominant) clonal lineage. Moreover, clonal similarity was high within compared to similarity between populations, that is, within each population clones differed only by single RAPD bands.

The genetic origin of this RAPD variation is not known. However, following Aagaard et al (1995) RAPD variation is most likely caused by noncoding and repetitive DNA, indicating neutral variation, whereas allozymes or coding DNA sequences represent variation in functional genes. As a consequence, because selection acts more strongly on genes encoding proteins, there is a supposedly weaker selective constraint on RAPD. This may explain why RAPD often detects more variation than techniques involving coding genes only (Rossi et al, 1998; Vandewoestijne and Baguette, 2002) and may be partly an explanation for the lower genetic variation reported in previous genetic studies of D. stevensoni (see above).

The present study was designed to investigate overall genotypic diversity in $D$. stevensoni by using RAPDs because allozymes were not a successful approach (see Rossi et al, 1998). However, since RAPD behave as dominant/recessive markers, bands can only be scored for presence/absence but homologous alleles cannot be assigned. Therefore, evaluating the Meselson effect is not possible from RAPD data. The latter effect can be tested by using DNA sequence data (see Mark Welch and Meselson, 2000; Schön and Martens, 2003).

\section{Clonal diversity in asexual populations}

Using published data to recalculate indices of clonal diversities from asexual populations from different species (the complete review with the recalculated data is available from KVD upon request) we found that irrespective the markers used, the indices $\left(N_{c}, N_{c} / n, G_{0}\right.$, $\left.G_{0} / N_{c}\right)$ vary within roughly similar ranges. The results also indicate that most asexuals $(80 \%)$ consist of multiclonal populations. The observed clonal variation in those asexual species has been mainly attributed to polyphyletic origins and asexual/sexual hybridisation (Little and Hebert, 1994; Schön et al, 2000 and references therein). For D. stevensoni the indices of clonal diversity follow the general trend of the literature (see Table 2) although it is assumed that only the accumulation of mutations is responsible for the observed variation, given that this species is supposed to be an ancient, obligatory asexual without a sexual root.
The clustering of clonal genotypes by region and the fact that clonal genotypes from the same populations tend to be genetically more similar to each other than to those from different populations, suggest that $D$. stevensoni 'clones' originate locally and do not seem to disperse much. This hypothesis fits well with the observation that $D$. stevensoni lacks dry-resistant eggs, which could be dispersed easily.

\section{Relationship between GPG and clonal diversity}

The present data suggest that the high ecological tolerance of $D$. stevensoni reported by Van Doninck et al (2002) is not associated with a single widespread 'clone', but involves at least four different local clonal genotypes that are ecologically highly tolerant. Hence, the prediction of the current GPG concept, that is that the wide ecological tolerance should be associated with a single widespread clonal lineage, is not corroborated here.

Recently, a series of papers on intraclonal genetic variation were published in a special issue of the Biological Journal of the Linnean Society (2003, 79). One of these by Loxdale and Lushai (2003) discusses the question 'what is a clone?'. Two definitions were given: firstly, a clone is an asexual lineage derived from a single mother; the second definition adds furthermore strict genetic, clonal fidelity. Loxdale and Lushai (2003), however, doubt the existence of strict clonal fidelity. Instead, a clone is a function of marker resolution and if sufficient regions of the genome are explored, each individual belonging to the same clone might eventually differ at some genomic regions. Keeping this in mind we propose to refine the GPG concept accordingly. A GPG is a single functional genotype producing a phenotype with a wide ecological tolerance but which may accumulate mutations in genomic regions that do not directly contribute to the phenotypic characters providing this broad ecological tolerance. In this view, the GPG concept should not be regarded as a genome-wide, fixed genotype, but rather as a specific genetic setup of a series of ecologically relevant genes.

In the absence of ecological differentiation between clones, the observed clonal diversity of $D$. stevensoni is then assumed to be the result of a balance between input of variation and stochastic extinction caused by genetic drift, the latter being determined by population size. This may be considered equivalent to the proposed GPG concept; that is, a broadly-adapted genotype that has accumulated neutral variation since the spread of the most recent advantageous mutation (Butlin et al, 1998).

Finally, the most challenging implication of the proposed GPG concept is that any test of the GPG based on variation at (nearly) neutral genetic markers cannot be conclusive.

\section{Conclusion}

The present work suggests that, (1) the asexual ostracod $D$. stevensoni shows genotypic variation within and between geographic populations; (2) no clonal lineage is dominant and widespread and (3) the amount and structuring of the clonal variation may still be consistent with the interpretation of $D$. stevensoni as a GPG, if the GPG is no longer considered as a genome wide phenomenon. 


\section{Acknowledgements}

We thank F Mezquita for the sampling of the Spanish population and Ken Irvine and Valery McCarthy for assistance in the field. P Van Doninck, R HellmissPeralta, C Claes and H Van Paesschen are acknowledged for their technical assitance with the graphics. This research was supported by a grant from the Flemish Institute for the Advancement of Scientific and Technological Research in Industry and Agriculture (IWT) to K Van Doninck.

\section{References}

Aagaard JE, Vollmer SS, Sorensen FC, Strauss SH (1995). Mitochondrial DNA products among RAPD profiles are frequent and strongly differentiated between races of Douglas fir. Mol Ecol 4: 441-447.

Black IV WC (1993). PCR with arbitrary primers: approach with care. Insect Mol Biol 2: 1-6.

Butlin RK, Schön I, Martens K (1998). Asexual reproduction in nonmarine ostracods. Heredity 81: 473-480.

Carvalho GR (1994). Evolutionary genetics of aquatic clonal invertebrates: concepts, problems and prospects. In: Beaumont AR (ed) Genetics and Evolution of Aquatic Organisms. Chapman \& Hall: London, pp 291-323.

Chaplin JA (1992). Variation in the mode of reproduction among individuals of the ostracod Candonocypris novaezelandiae. Heredity 68: 411-424.

Chaplin JA, Havel JE, Hebert PDN (1994). Sex and ostracods. Trends Ecol Evol 9: 435-439.

Chen CA, Wei NV, Dai C-F (2002). Genotyping the clonal population structure of a gorgonian coral, Junceella fragilis (Anthozoa: Octocorallia: Ellisellidae) from Lanyu, Taiwan, using Simple Sequence Repeats in Ribosomal Intergenic Spacer. Zool Stud 41: 295-302.

Coffroth MA, Lasker HR (1998). Population structure of a clonal gorgonian coral: the interplay between clonal reproduction and disturbance. Evolution 52: 379-393.

Fukatsu T, Ishikawa H (1994). Differentiation of aphid clones by arbitrarily primed polymerase chain reaction (AP-PCR) DNA fingerprinting. Mol Ecol 3: 187-192.

Gandolfi A, Bonilauri P, Rossi V, Menozzi P (2001). Intraindividual and intraspecies variability of ITS1 sequences in the ancient asexual Darwinula stevensoni (Crustacea: Ostracoda). Heredity 87: 449-455.

Guirao P, Moya A, Cenis JL (1995). Optimal use of Random Amplified Polymorphic DNA in estimating the genetic relationship of four major Meloidogyne spp. Genetics 85: 547-551.

Havel JE, Hebert PDN (1993). Clonal diversity in parthenogenetic ostracods. In: McKenzie KG, Jones PJ (eds) Ostracoda in the Earth and Life Sciences. A.A. Balkema: Rotterdam, pp 353-368.

Hughes RN (1989). A Functional Biology of Clonal Animals. Chapman \& Hall: London.

Jacobsen R, Forbes VE, Skovgaard O (1996). Genetic population structure of the prosobranch snail Potamopyrgus antipodarum (Gray) in Denmark using PCR-RAPD fingerprints. Proc Roy Soc London B 263: 1065-1070.

Little TJ, Hebert PDN (1994). Abundant asexuality in tropical freshwater ostracodes. Heredity 73: 549-555.

Loxdale HD, Lushai G (2003). Rapid changes in clonal lines: the death of a 'sacred cow'. Biol J Linn Soc 79: 3-16.

Lynch M (1984). Destabilizing hybridization, general-purpose genotypes and geographic parthenogenesis. Quart Rev Biol 59: $257-290$.

Lynch M, Spitze K, Crease TJ (1989). The distribution of life history variation in the Daphnia pulex complex. Evolution 43: 1724-1736.
Mark Welch D, Meselson M (2000). Evidence for the evolution of Bdelloid Rotifers without sexual reproduction or genetic exchange. Science 288: 1211-1214.

Martens K (1998). Sex and Parthenogenesis: Evolutionary Ecology of Reproductive Modes in Non-marine Ostracods. Backhuys Publishers: Leiden.

Martens K, Rossetti G, Horne DJ (2003). How ancient are ancient asexuals? Proc Roy Soc London B 270: 723-729.

Nei M (1973). Analysis of gene diversity in subdivided populations. Proc Natl Acad Sci USA 70: 3321-3323.

Nei M, Li W-H (1979). Mathematical model for studying genetic variation in terms of restriction endonucleases. Proc Natl Acad Sci USA 76: 5269-5273.

Parker ED (1979). Ecological implications of clonal diversity in parthenogenetic morphospecies. Am Zool 19: 753-762.

Parker ED, Niklasson M (1998). Genetic structure and evolution in parthenogenetic animals. In: Singh R, Krimbas C (eds) Evolutionary Genetics from Molecules to Morphology. University Press: Cambridge.

Rohlf FJ (1993). NT-SYS pc version 1.80. Applied Biostatistics, Inc.: Setauket.

Rossetti G, Martens K (1998). Taxonomic revision of the Recent and Holocene representatives of the Family Darwinulidae (Crustacea, Ostracoda), with a description of three new genera. Bull $k$ bel Inst Natuurw Biol 68: 55-110.

Rossi V, Giordano P, Menozzi P (1993). Genetic variability in parthenogenetic populations of Heterocyrpis incongruens (Ostracoda). In: McKenzie KG, Jones PJ (eds) Ostracoda in the Earth and Life Sciences. A.A. Balkema: Rotterdam, pp 369-383.

Rossi V, Schön I, Butlin RK, Menozzi P (1998). Clonal genetic diversity. In: Martens $\mathrm{K}$ (ed) Sex and Parthenogenesis: Evolutionary Ecology of Reproductive Modes in Non-marine Ostracods. Backhuys Publishers: Leiden, pp 257-274.

Rossi V, Todeschi EBA, Gandolfi A, Invidia M, Menozzi P (2002). Hypoxia and starvation tolerance in individuals from a riverine and a lacustrine population of Darwinula stevensoni (Crustacea: Ostracoda). Arch Hydrobiol 154: 151-171.

Schizas NV, Street GT, Coull BC, Chandler GT, Quattro JM (1997). An efficient DNA extraction method for small metazoans. Mol Mar Biol Biotechnol 6: 381-383.

Schön I, Butlin RK, Griffiths HI, Martens K (1998). Slow molecular evolution in an ancient asexual ostracod. Proc Roy Soc London B 265: 235-242.

Schön I, Gandolfi A, Masso ED, Rossi V, Griffiths HI, Martens K et al (2000). Persistence of asexuality through mixed reproduction in Eucypris virens (Crustacea, Ostracoda). Heredity 84: 161-169.

Schön I, Martens K (2003). No slave to sex. Proc Roy Soc London B 270: 827-833.

Stoddart JA (1983). A genotypic diversity measure. J Hered 74: 489-490.

Straub EB (1952). Mikropaläontologische Untersuchungen im Tertiär zwischen Ehingen und Ulm a. d. Donau. Geol Jb 66 : 433-523.

Van Doninck K, De Bruyn L, Schön I, Martens K (2002). A general purpose genotype in an ancient asexual. Oecologia 132: $205-212$

Van Opijnen T, Breeuwer JAJ (1999). High temperatures eliminate Wolbachia, a cytoplasmic incompatibility inducing endosymbiont, from the two-spotted spider mite. Exp Appl Acarol 23: 871-881.

Vandewoestijne S, Baguette M (2002). The genetic structure of endangered populations in the Cranberry Fritillary, Boloria aquilonaris (Lepidoptera, Nymphalidae): RAPDs vs allozymes. Heredity 89: 439-445.

Vrijenhoek RC (1998). Clonal organisms and the benefits of sex. In: Carvalho G (ed) Advances in Molecular Ecology. IOS Press: Amsterdam, pp 151-172. 
Williams JGK, Kubelik AR, Livak KJ, Rafalski JA, Tingey SV (1990). DNA polymorphisms amplified by arbitrary primers are useful as genetic markers. Nucl Acids Res 18: 6531-6535. Yap IV, Nelson RJ (1996). WinBoot: a program for performing bootstrap analysis of binary data to determine the confidence limits of UPGMA-based dendrograms. IRRI: Manila Philippines. http://www.irri.org/science/software/winboot.asp.

Yeh FC, Boyle T (1997). Popgene version 1.2: Microsoft Window-based software for population genetic analysis. http:// www.ualberta.ca/ fyeh/. 